\title{
Lack of association of two common polymorphisms on 9p2I with risk of coronary heart disease and myocardial infarction; results from a prospective cohort study
}

\author{
Abbas Dehghan ${ }^{1}$, Mandy van Hoek ${ }^{2}$, Eric JG Sijbrands², Ben A Oostra ${ }^{3}$, \\ Albert Hofman ${ }^{1}$, Cornelia $M$ van Duijn ${ }^{1}$ and Jacqueline CM Witteman*1
}

\author{
Address: ${ }^{1}$ Department of Epidemiology, Erasmus Medical Center, Rotterdam, the Netherlands, ${ }^{2}$ Department of Internal Medicine, Erasmus \\ Medical Center, Rotterdam, the Netherlands and ${ }^{3}$ Department of Clinical Genetics, Erasmus Medical Center, Rotterdam, the Netherlands \\ Email: Abbas Dehghan - a.dehghan@erasmusmc.nl; Mandy van Hoek - m.vanhoek@erasmusmc.nl; \\ Eric JG Sijbrands - e.sijbrands@erasmusmc.nl; Ben A Oostra - b.oostra@erasmusmc.nl; Albert Hofman - a.hofman@erasmusmc.nl; \\ Cornelia M van Duijn - c.vanduijn@erasmusmc.nl; Jacqueline CM Witteman* - J.witteman@erasmusmc.nl \\ * Corresponding author
}

Published: 16 October 2008

BMC Medicine 2008, 6:30 doi:10.1186/174I-7015-6-30

This article is available from: http://www.biomedcentral.com/I74I-70I5/6/30

(c) 2008 Dehghan et al; licensee BioMed Central Ltd.

This is an Open Access article distributed under the terms of the Creative Commons Attribution License (http://creativecommons.org/licenses/by/2.0), which permits unrestricted use, distribution, and reproduction in any medium, provided the original work is properly cited.
Received: 31 January 2008

Accepted: 16 October 2008

\begin{abstract}
Background: Recent genome wide association (GWA) studies identified two Single Nucleotide Polymorphisms (SNP) (rs 10757278 and rs 10757274 ) in the region of the CDK2NA and CDK2NB genes to be consistently associated with the risks of coronary heart disease (CHD) and myocardial infarction (MI). We examined the SNPs in relation to the risk of CHD and $\mathrm{MI}$ in a large population based study of elderly population.
\end{abstract}

Methods: The Rotterdam Study is a population-based, prospective cohort study among 7983 participants aged 55 years and older. Associations of the polymorphisms with CHD and MI were assessed by use of Cox proportional hazards analyses.

Results: In an additive model, the age and sex adjusted hazard ratios (HRs) (95\% confidence interval) for CHD and MI were $1.03(0.90,1.18)$ and $0.94(0.82,1.08)$ per copy of the G allele of rs I0757274. The corresponding HRs were I.03 $(0.90, \mathrm{I} . \mathrm{I})$ and $0.93(0.8 \mathrm{I}, \mathrm{I} .06)$ for the $\mathrm{G}$ allele of rs 10757278. The association of the SNPs with CHD and MI was not significant in any of the subgroups of CHD risk factors.

Conclusion: we were not able to show an association of the studied SNPs with risks of CHD and MI. This may be due to differences in genes involved in the occurrence of CHD in young and older people.

\section{Background}

It has been considered for long that genes play a substantial role in susceptibility to coronary heart disease (CHD) [1]. Up to now, a limited number of these genes have been identified through the candidate gene approach and genome wide linkage studies. Recently a number of genome wide association (GWA) studies have identified several genetic variants on chromosome 9p21 associated with the risk of CHD. McPherson et al. found a Single Nucleotide Polymorphism (SNP), rs10757274, on chromosome 9p21 associated with the risk of CHD [2]. Helgadottir et al. found a close-by SNP, rs10757278, in the 
same 9p21 region associated with the risk of myocardial infarction (MI) [3]. These findings were followed by another GWA study by Samani et al [4], which found rs1333049 to be associated with the risk of coronary artery disease [4]. All three SNPs are located within the same Linkage Disequilibrium (LD) block on chromosome 9 approximately 22 million base pairs from the $9 \mathrm{p}$ telomere, adjacent to two tumor suppressor genes, CDKN2A and $\mathrm{CDKN} 2 \mathrm{~B}$. These genes are involved in regulation of cell proliferation. Abnormal proliferation is one of the characteristics of atherosclerosis, one of the pathological features of CHD and MI [5].

To date, the findings are replicated in several case-control studies comprising 12285 cases and 23184 controls and two cohort studies comprising 22056 subjects [6-10]. These replications has made this locus one of the best replicated findings for genetic susceptibility to cardiovascular diseases. Though this findings are promising, they will be of more clinical worth if translated to older patients who constitute a larger part of the patients. We chose to study rs10757278 and rs10757274 because they were most strongly and consistently associated with CHD and MI in GWA studies. The leading SNP of the study by Samani et al, rs1333049, is in the same LD block with rs 10757278 and contributes to the same haplotype alleles. We attempted to replicate the association in the Rotterdam Study, a population-based cohort study among older subjects, but found no association.

\section{Methods}

\section{Study Population}

The study was conducted within the framework of the Rotterdam Study, an ongoing prospective, populationbased cohort study on determinants of a number of chronic diseases. The Rotterdam Study has been described in detail elsewhere [11]. In brief, all inhabitants of Ommoord, a district of Rotterdam in the Netherlands, who were 55 years or over, were invited to participate in this study. Of all 10275 eligible individuals, 7983 agreed to participate $(78 \%)$. Written informed consent was obtained from all participants and the Medical Ethics Committee of the Erasmus Medical Center approved the study.

\section{Baseline measurements}

The baseline examinations took place from 1990-1993. Participants were visited at home for an interview. Information on current health status, medical history, use of medication, and smoking status were obtained during the interview. The interview was followed by two visits at the research center for blood sampling and further examinations.
At baseline, participants were asked whether they have ever experienced a heart attack. A 12-lead electrocardiogram (ECG) was stored digitally and analyzed by using the Modular ECG Analysis System (MEANS). Myocardial infarction found on ECG was based on criteria partly derived from the Minnesota code. A history of myocardial infarction was considered present in case of a self-report of myocardial infarction confirmed by ECG or additional clinical information, or the presence of an ECG characteristic of prior myocardial infarction $[12,13]$.

\section{Genotyping}

Genomic DNA was extracted from leucocytes following standard procedures. Participants were genotyped for rs10757274 and rs10757278. Genotypes were determined in our study population in 2-ng genomic DNA by use of pre-designed TaqMan SNP genotyping assay (Assay ID C_26505812_10 and C_11841860_10, respectively; Applied Biosystems, Foster City, CA). Reactions were performed with the Taqman Prism 7900 HT 384 wells format.

\section{Follow up}

Follow up for clinical events started at baseline and follow up examinations were carried out periodically in 19951996, 1997-1999, and 2002-2004. Participants were continuously monitored for fatal and nonfatal cardiovascular events through automated linkage with files from general practitioners and pharmacies working in the study district of Ommoord. In addition, all medical records of the participants under the care of general practitioners outside the study area were checked annually. Two research physicians independently coded all reported events according to the International Classification of Diseases, 10th edition (ICD-10). Codes on which the research physicians disagreed were discussed to reach consensus. Finally, a medical expert in cardiovascular disease, whose judgment was considered final, reviewed all events. Information on vital status was obtained regularly from municipal health authorities in Rotterdam. For the present study, follow up data were available until October $1,2005$.

\section{Incident coronary heart disease and myocardial infarction} To identifying incident myocardial infarction and coronary heart disease, we collected information from baseline (1990 - 1993) until January 1, 2005. Fatal or nonfatal MI reported by general practitioners in the research area, letters from medical specialists and discharge reports for hospitalized patients were the sources of information used. Two research physicians coded the events independently and in case of disagreement the consensus was made ina separate session. Finally a specialist whose judgment was considered final verified the coding. We defined incident MI as fatal or non fatal MI (ICD-10 code I21). Inci- 
dent CHD was defined as fatal or nonfatal myocardial infarction (ICD-10 code I21), coronary artery bypass grafting (CABG), and percutaneous transluminal coronary angioplasty (PTCA).

\section{Population for analysis}

The SNPs, rs 10757274 and rs 10757278 were genotyped in 6251 and 6265 out of 7129 participants who visited the research center at baseline.

\section{Statistical analysis}

Genotype frequencies were tested for Hardy-Weinberg equilibrium with a chi-square test using The Hardy-Weinberg package for $\mathrm{R}$ http://www.r-project.org. To compare the baseline characteristics between healthy subjects and those who experienced CHD or MI, we used chi-square for categorical variables and ANOVA for continuous variables.

A Cox regression analysis was used to assess the association of SNPs with incident CHD and MI. The proportional hazards assumption was validated by the use of a timedependent variable to check increasing or decreasing trends in the hazard ratio (HR) over time. The basic model was adjusted for age and sex. The multivariate adjusted model was additionally adjusted for BMI, systolic and diastolic blood pressure, total cholesterol, HDL cholesterol, smoking, and diabetes.

To examine whether the effect of SNPs vary by the level of other risk factors we performed the analysis stratified by age, sex, family history of cardiovascular disease, HDL cholesterol, diabetes, hypertension, smoking, and history of CHD. For smoking, participants were categorized to never, former, and current smokers. For hypertension and diabetes, participants were categorized into those with and without the condition. History of CHD was defined as a history of MI, percutaneous transluminal coronary angioplasty (PTCA) or coronary artery bypass grafting (CABG). For other risk factors, the population was divided into two equal subgroups by use of the median. All statistical analyses were performed with the use of SAS, version 8 .

\section{Results}

Table 1 shows the baseline characteristics of the studied population. CHD and MI cases were significantly older, and more often male, hypertensive, diabetic, and smoker than subjects without these conditions. Moreover, systolic blood pressure, and total cholesterol were significantly higher and HDL cholesterol was significantly lower in CHD and MI cases. We found none of these characteristics to be significantly associated with the studied SNPs.

During follow-up, 588 participants developed CHD, of which 412 had experienced an MI. The mean follow-up time was 9.5 years for CHD (incidence rate 9.9 per 1000 person years) and 9.5 years for MI (incidence rate 6.9 per 1000 person years). Mean (standard deviation) age of onset was 68.6 (7.4) for CHD and 70.3 (7.8) years for MI. We examined the associations of rs 10757274 and rs 10757278 with risks of CHD and MI (table 2, 3 and 4). None of the SNPs were significantly associated with the risk of CHD or MI. The age and sex adjusted HR (95\% confidence interval [CI]) for $\mathrm{CHD}$ and $\mathrm{MI}$ were 1.03 $(0.90,1.18)$ and $0.94(0.82,1.08)$ per copy of $G$ allele of rs 10757274, respectively. The corresponding HRs were $1.03(0.90,1.18)$ and $0.93(0.81,1.06)$ per copy of $G$ allele of rs10757278.

We repeated the analysis with incident cases limited to those occurred before age 70. Age and sex adjusted HR $(95 \% \mathrm{CI})$ for CHD and MI were $1.00(0.97,1.04)$ and $0.90(0.74,1.09)$ per copy of $G$ allele of rs 10757274 . The corresponding HRs for MI were $1.00(0.96,1.04)$, and $0.91(0.75,1.10)$ per copy of $G$ allele of rs10757278.

Table I: Baseline characteristics of all participants, and of cases with incident MI and incident CHD

\begin{tabular}{|c|c|c|c|c|c|}
\hline Characteristics & All participants & MI cases & $P$ value & $\mathrm{CHD}$ cases & $P$ value \\
\hline Number & 6251 & 412 & - & 588 & - \\
\hline Age, mean (SD), y & $69.5(9.1)$ & $70.3(7.8)$ & 0.02 & $68.6(7.4)$ & 0.23 \\
\hline Men, $(\%)$ & 40.4 & 58.7 & $<0.001$ & 61.2 & $<0.001$ \\
\hline Body mass index, mean (SD), kg/m2 & $26.3(3.7)$ & $26.3(3.5)$ & 0.29 & $26.3(3.4)$ & 0.07 \\
\hline Waist circumference, mean (SD), cm & $90.5(11.2)$ & $91.9(10.0)$ & 0.89 & $92.2(10.2)$ & 0.33 \\
\hline Systolic blood pressure, mean (SD), mm Hg & $139.3(22.2)$ & $142.3(21.9)$ & 0.01 & $14 \mid .3(21.7)$ & 0.004 \\
\hline Diastolic blood pressure, mean (SD), $\mathrm{mm} \mathrm{Hg}$ & $73.7(11.5)$ & $73.7(11.5)$ & 0.77 & $73.7(11.3)$ & 0.38 \\
\hline Hypertension, (\%) & 34.3 & 40.6 & 0.006 & 40.2 & 0.002 \\
\hline Diabetes, (\%) & 10.5 & 17.0 & $<0.001$ & 15.8 & $<0.001$ \\
\hline Total cholesterol, mean (SD), mmol/L & $6.6(1.2)$ & $6.9(1.2)$ & $<0.001$ & $6.9(1.2)$ & $<0.001$ \\
\hline HDL cholesterol, mean (SD), mmol/L & I.3 (0.4) & $1.2(0.3)$ & $<0.001$ & $1.2(0.3)$ & $<0.001$ \\
\hline Current smoker, (\%) & 22.8 & 26.0 & $<0.001$ & 27.1 & $<0.001$ \\
\hline Former smoker, (\%) & 41.5 & 49.5 & & 51.5 & \\
\hline
\end{tabular}


Table 2: The age and sex adjusted and multivariate adjusted association of the SNPs with incident CHD and MI

\begin{tabular}{ccccccc}
\hline & & Allele & All participants & Incident cases & Age and sex adjusted & Multivariate adjusted HR* \\
\hline \multirow{2}{*}{ CHD } & rsI0757274 & G & 45.8 & 45.5 & $1.03(0.90,1.18)$ & $1.00(0.87,1.15)$ \\
& rsI0757278 & G & 44.9 & 42.7 & $1.03(0.90,1.18)$ & $1.00(0.87,1.15)$ \\
MI & rsI0757274 & G & 45.8 & 43.8 & $0.94(0.82,1.08)$ & $0.97(0.84,1.11)$ \\
& rs10757278 & G & 44.9 & 42.7 & $0.93(0.81,1.06)$ & $0.95(0.83,1.10)$ \\
\hline
\end{tabular}

* adjusted for age, sex, BMI, serum total and HDL cholesterol, smoking, diabetes, systolic and diastolic blood pressure.

$\mathrm{Cl}=$ Confidence interval, $\mathrm{CHD}=$ Coronary heart disease, $\mathrm{MI}=$ Myocardial infarction

To investigate whether any of the covariates affect the relation of SNPs with CHD and MI, we repeated the analysis in subgroups of age, sex, family history of cardiovascular disease, HDL cholesterol, diabetes, hypertension, smoking, and history of CHD (figure 1). The association was not significant in any of the studied subgroups and no significant interaction was found.

\section{Discussion}

Our main goal was to replicate the results of recent GWA studies on CHD and MI in a population based study. Two SNPs, rs10757274 and rs10757278, which were most consistently and strongly associated with the risk of CHD in GWA studies were studied. We not only found no significant association between these two SNPs and the risks of CHD and MI, but also found, however non-significant, an inverse direction for the risk. We also did not find an association in subgroups of cardiovascular risk factors.

Different approaches have been used in recent decades to discover causal genes for cardiovascular diseases. A novel approach is the GWA study which searches large part of the genome for predisposing variants. Contrary to the formerly common approach, the candidate gene study, the GWA study is a hypothesis free approach, i.e. it holds no prior assumption on the location of predisposing genes. As an advantage, this approach promises a more comprehensive understanding of the causal genes. However, this method is liable to false positive findings. Hence, GWA studies always need to be replicated in independent samples to confirm their findings.

Our study had sufficient power to detect effect sizes as shown in the published studies. In an additive model and for a SNP with a minor allele frequency of 0.45 (lowest minor allele frequency: 0.43 , shown in Icelandic population A [3]), our study had more than $80 \%$ power to detect a relative risk of 1.15 for CHD (the lowest effect: 1.18, shown in ARIC study [2]) and 1.23 for MI (the lowest effect: 1.25 , shown in the Iceland population A) [14].

We did not find any of the SNPs to be associated with the risk of CHD in our study. One legitimate conjecture for the inconsistency of our results with former studies may be heterogeneity of the effect. Compared to the Rotterdam Study, most of the studied populations comprised young CHD or MI patients. If the risk allele on chromosome 9p21 invokes only early onset of CHD, the effect in older subjects may not be large enough to be found in our study. Therefore, our negative finding may point to a heterogeneity of effect by age. In agreement with this conjecture, Helgadottir et al. showed that the association was stronger when only those with early onset MI were considered [3]. However, we failed to find an evidence of age affecting the association in our data. The strength of the association did not change materially when we limited the incident cases to those developed CHD or MI before age 70 . Moreover, the strength of the association was not

Table 3: The age and sex adjusted and multivariate adjusted association of the SNPs with incident CHD

\begin{tabular}{|c|c|c|c|}
\hline & Participants (cases) & Age and sex adjusted & Multivariate adjusted $*$ \\
\hline \multicolumn{4}{|c|}{ rs 10757274} \\
\hline AA & $1834(184)$ & Reference & Reference \\
\hline AG & $3107(273)$ & $0.89(0.74,1.07)$ & $0.90(0.74,1.09)$ \\
\hline GG & $1310(131)$ & $1.00(0.80,1.26)$ & $0.99(0.78,1.25)$ \\
\hline \multicolumn{4}{|c|}{ rs 10757278} \\
\hline $\mathrm{AA}$ & $1909(188)$ & Reference & Reference \\
\hline AG & $3097(269)$ & $0.90(0.74,1.08)$ & $0.90(0.74,1.09)$ \\
\hline GG & 1264 (127) & $1.01(0.81,1.27)$ & $1.00(0.79,1.26)$ \\
\hline
\end{tabular}

* adjusted for age, sex, BMI, serum total and HDL cholesterol, smoking, diabetes, systolic and diastolic blood pressure.

$\mathrm{Cl}=$ Confidence interval, $\mathrm{CHD}=$ Coronary heart disease, $\mathrm{MI}=$ Myocardial infarction 
Table 4: The age and sex adjusted and multivariate adjusted association of the SNPs with incident MI

\begin{tabular}{|c|c|c|c|}
\hline & Participants (cases) & Age and sex adjusted & Multivariate adjusted $*$ \\
\hline \multicolumn{4}{|c|}{ rs10757274 } \\
\hline $\mathrm{AA}$ & $1832(133)$ & Reference & Reference \\
\hline AG & $3106(197)$ & $0.90(0.72,1.12)$ & $0.94(0.75,1.19)$ \\
\hline GG & $1309(82)$ & $0.89(0.67,1.17)$ & $0.94(0.70,1.25)$ \\
\hline \multicolumn{4}{|c|}{ rs10757278 } \\
\hline AA & 1907 (139) & Reference & Reference \\
\hline AG & $3096(187)$ & $0.85(0.68,1.06)$ & $0.89(0.71,1.12)$ \\
\hline GG & $1263(80)$ & $0.88(0.67,1.16)$ & $0.92(0.70,1.23)$ \\
\hline
\end{tabular}

* adjusted for age, sex, BMI, serum total and HDL cholesterol, smoking, diabetes, systolic and diastolic blood pressure.

$\mathrm{Cl}=$ Confidence interval, $\mathrm{CHD}=$ Coronary heart disease, $\mathrm{MI}=$ Myocardial infarction

Age $<68.7$ years

Age $>68.7$ years

Men

Women

No family History

With family History

HDL cholesterol $=<1.3$

HDL cholesterol $>1.3$

No diabetes

With Diabetes

No HTN

With HTN

Current smoker

former smoker

never smoker

CHD History

No CHD history

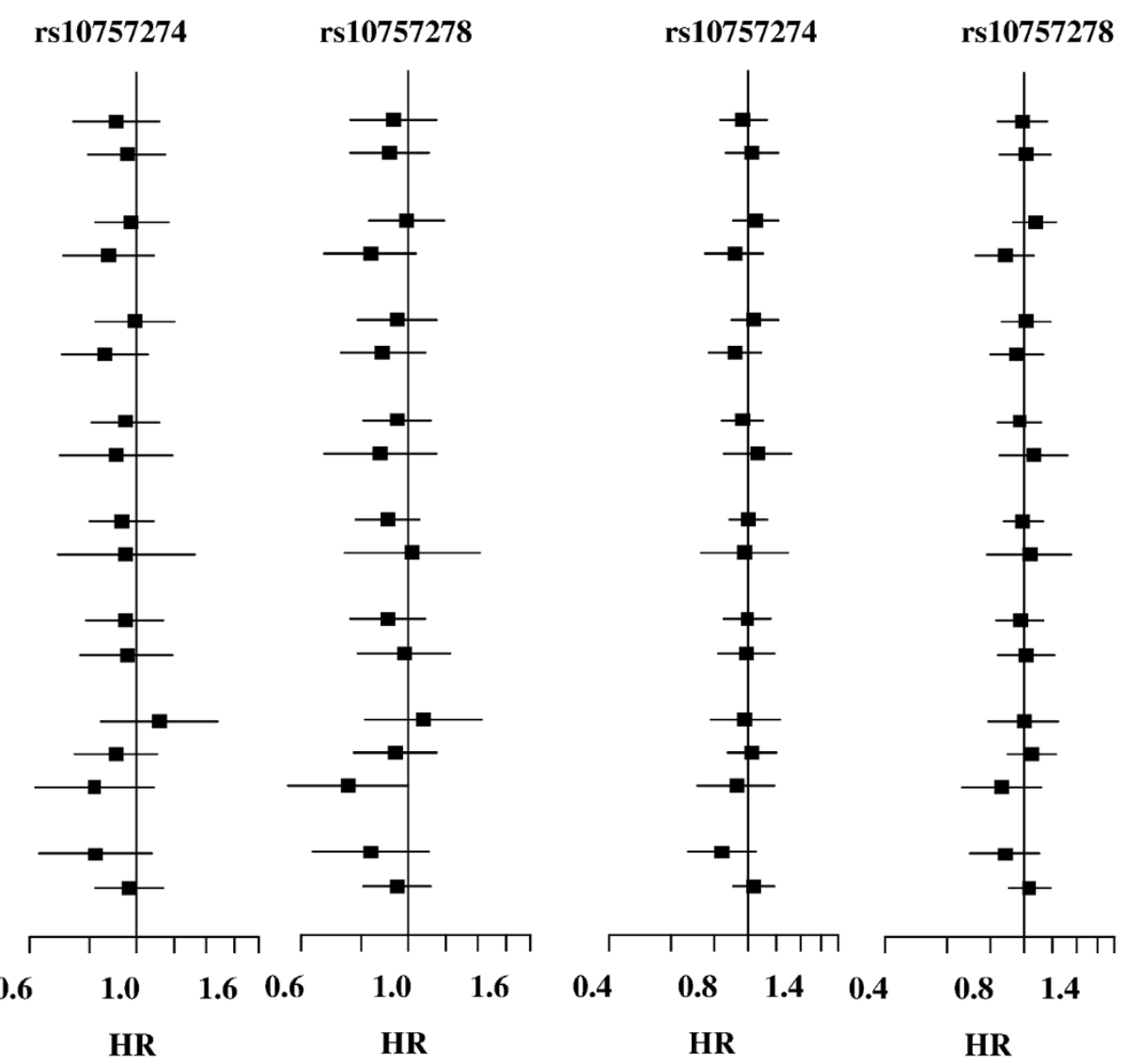

Figure I

Association of rs 10757274 and rs 10757278 with CHD and MI in subgroups of cardiovascular risk factors. The solid squares centered are on the hazard ratio estimate and scaled proportional to the sample size. Horizontal bars show the $95 \%$ confidence interval. 
significantly different in age subgroups (figure 1). We emphasis that our study may be underpowered to detect the effect of age on the association. It is noteworthy that the heterogeneity of effect has a particular clinical and public health impact. In Western countries the majority of morbidity and mortality from CHD occurs in elderly people. In the Netherlands, $74 \%$ of men and $91 \%$ women who experience fatal MI are older than 65 years http://stat line.cbs.nl/statweb/. The fact that CHD is less common in younger population implies that these subjects are not a good representative of the general population of CHD patients. Therefore, caution should be taken in generalizing the results of the published studies to an elderly population.

One may also speculate that those carrying the risk allele had developed CHD at early age and were excluded at the baseline in our study. If this is true, the prevalence of risk allele should be higher in those who had a history of CHD at baseline i.e. prevalent cases of CHD. To examine this issue, we studied the association of the SNPs with prevalent CHD cases but found no association (data not shown). Moreover, the frequencies of the alleles in our population were high and comparable to former studies making selection bias unlikely.

Previous studies mainly employed standard case-control association studies. Our study has the advantage of employing a different approach, the prospective study in a large population based sample. One potential limitation of our study is that the participants were not fully followed and healthy subjects who carry the risk allele may later develop the disease.

\section{Conclusion}

In conclusion, we showed that the studied SNPs are not major players in development of CHD in the elderly population. Our negative finding offer a new perspective on 9p21 SNPs and shows that the association does not hold for all CHD cases. The lack of association may be due to the difference in genes involved in the development of CHD in young and older people. Individualized preventive measures and therapies constitute a major long-term goal of GWA studies. Heterogeneity of the effect, therefore, has substantial public health impact and needs to be acknowledged.

\section{Abbreviations}

GWA: Genome Wide Association; SNP: Single Nucleotide Polymorphism; CHD: Coronary Heart Disease; MI: Myocardial Infarction; HR: Hazard Ratio; LD: Linkage Disequilibrium; ECG: Electrocardiogram; ICD: International Classification of Diseases; CABG: Coronary Artery Bypass Grafting; PTCA: Percutaneous Transluminal Coronary Angioplasty.

\section{Competing interests}

The authors declare that they have no competing interests.

\section{Authors' contributions}

$\mathrm{AD}$ contributed to the design of this study, performed the statistical analysis, and drafted the manuscript. $\mathrm{MvH}$, EJGS, and JCMW contributed to the conception and the design of this study and critically revised the manuscript. $\mathrm{AH}, \mathrm{CvD}$, and $\mathrm{BO}$ has made substantial contributions to the interpretation of results, and critically revised the manuscript. All authors have read and approved the final manuscript.

\section{Acknowledgements}

The Rotterdam Study is supported by the Erasmus Medical Center and Erasmus University Rotterdam; the Netherlands Organization for Scientific Research; the Netherlands Organization for Health Research and Development (ZonMw); the Research Institute for Diseases in the Elderly; the Ministry of Education, Culture and Science; the Ministry of Health, Welfare and Sports; the European Commission; and the Municipality of Rotterdam. Dr. Abbas Dehghan is supported by a scholarship from Hormozgan University of Medical Science, Bandar Abbas, Iran.

\section{References}

I. Slack J, Evans KA: The increased risk of death from ischaemic heart disease in first degree relatives of 121 men and 96 women with ischaemic heart disease. J Med Genet 1966, 3(4):239-257.

2. McPherson R, Pertsemlidis A, Kavaslar N, Stewart A, Roberts R, Cox DR, Hinds DA, Pennacchio LA, Tybjaerg-Hansen A, Folsom AR, et al:: A common allele on chromosome 9 associated with coronary heart disease. Science 2007, 3 | 6(5830): |488- I 49| .

3. Helgadottir A, Thorleifsson G, Manolescu A, Gretarsdottir S, Blondal T, Jonasdottir A, Sigurdsson A, Baker A, Palsson A, Masson G, et al.: A common variant on chromosome $9 \mathrm{p} 2 \mathrm{I}$ affects the risk of myocardial infarction. Science 2007, 316(5830): 149|-1493.

4. Samani NJ, Erdmann J, Hall AS, Hengstenberg C, Mangino M, Mayer B, Dixon RJ, Meitinger T, Braund P, Wichmann HE, et al:: Genomewide association analysis of coronary artery disease. N Engl J Med 2007, 357(5):443-453.

5. Rodriguez I, Coto E, Reguero JR, Gonzalez P, Andres V, Lozano I, Martin M, Alvarez V, Moris C: Role of the CDKNIA/p2I, CDKNIC/p57, and CDKN2A/p I 6 genes in the risk of atherosclerosis and myocardial infarction. Cell Cycle 2007, 6(5):620-625.

6. Abdullah KG, Li L, Shen GQ, Hu Y, Yang Y, Mackinlay KG, Topol EJ, Wang QK: Four SNPS on Chromosome 9p2I Confer Risk to Premature, Familial CAD and MI in an American Caucasian Population (GeneQuest). Ann Hum Genet 2008.

7. Chen Z, Qian Q, Ma G, Wang J, Zhang X, Feng Y, Shen C, Yao Y: A common variant on chromosome $9 \mathrm{p} 2 \mathrm{I}$ affects the risk of early-onset coronary artery disease. Mol Biol Rep 2008.

8. Shen GQ, Rao S, Martinelli N, Li L, Olivieri O, Corrocher R, Abdullah KG, Hazen SL, Smith J, Barnard J, et al.: Association between four SNPs on chromosome 9p2I and myocardial infarction is replicated in an Italian population. J Hum Genet 2008, 53(2): $144-150$.

9. Shen GQ, Li L, Rao S, Abdullah KG, Ban JM, Lee BS, Park JE, Wang QK: Four SNPs on chromosome 9p2I in a South Korean population implicate a genetic locus that confers high cross-race risk for development of coronary artery disease. Arterioscler Thromb Vasc Biol 2008, 28(2):360-365.

10. Schunkert H, Gotz A, Braund P, McGinnis R, Tregouet DA, Mangino M, Linsel-Nitschke P, Cambien F, Hengstenberg C, Stark K, et al: Repeated replication and a prospective meta-analysis of the association between chromosome 9p21.3 and coronary artery disease. Circulation 2008, II 7(13): $1675-1684$.

11. Hofman A, Breteler MM, van Duijn CM, Krestin GP, Pols HA, Stricker BH, Tiemeier H, Uitterlinden AG, Vingerling JR, Witteman JC: The 
Rotterdam Study: objectives and design update. Eur J Epidemiol 2007, 22(I I):8I9-829.

12. Meer IM van der, Bots ML, Hofman A, del Sol Al, Kuip DA van der, Witteman JC: Predictive value of noninvasive measures of atherosclerosis for incident myocardial infarction: the Rotterdam Study. Circulation 2004, I09(9): 1089-1094.

13. de Bruyne MC, Mosterd A, Hoes AW, Kors JA, Kruijssen DA, van Bemmel JH, Hofman A, Grobbee DE: Prevalence, determinants, and misclassification of myocardial infarction in the elderly. Epidemiology 1997, 8(5):495-500.

14. Gauderman WJ: Sample size requirements for matched casecontrol studies of gene-environment interaction. Stat Med 2002, 2 I (I):35-50.

\section{Pre-publication history}

The pre-publication history for this paper can be accessed here:

http://www.biomedcentral.com/1741-7015/6/30/prepub

Publish with Bio Med Central and every scientist can read your work free of charge

"BioMed Central will be the most significant development for disseminating the results of biomedical research in our lifetime. "

Sir Paul Nurse, Cancer Research UK

Your research papers will be:

- available free of charge to the entire biomedical community

- peer reviewed and published immediately upon acceptance

- cited in PubMed and archived on PubMed Central

- yours - you keep the copyright

Submit your manuscript here:

http://www.biomedcentral.com/info/publishing_adv.asp 